\title{
Scanning Coherent Scattering Methods for Actinic EUV Mask Inspection
}

\author{
Y. Ekinci*a, P. Helfenstein ${ }^{\mathrm{a}}$, R. Rajeev ${ }^{\mathrm{a}}$, I.Mochi ${ }^{\mathrm{a}}$, I. Mohacsi $^{\mathrm{a}}$, J. Gobrecht ${ }^{\mathrm{a}}$, and S. Yoshitake ${ }^{\mathrm{b}}$

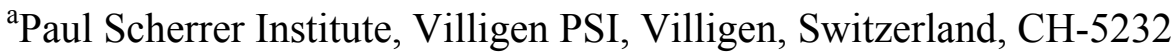 \\ ${ }^{\mathrm{b}}$ NuFlare Technology, Inc., 8-1 Shinsugita-cho, Isogo-ku, Yokohama 235-8522, Japan
}

\begin{abstract}
Actinic mask inspection for EUV lithography with targeted specifications of resolution, sensitivity, and throughput remains a big hurdle for the successful insertion of EUVL into high volume manufacturing and effective solutions are needed to address this. We present a method for actinic mask inspection based on scanning coherent scattering microscopy. In this method, the mask is scanned with an EUV beam of relatively small spot size and the scattered light is recorded with a pixel detector. Customized algorithms reconstruct the aerial image by iteratively solving the phaseproblem using over-determined diffraction data gathered by scanning across the specimen with a finite illumination. This approach provides both phase and amplitude of actinic aerial images of the mask with high resolution without the need to use high NA (numerical aperture) lenses. Futher, we describe a reflective mode EUV mask scanning lensless imaging tool (RESCAN), which was installed at the XIL-II beamline and later at the SIM beamline of the Swiss Light Source and show reconstructed aerial images down to $10 \mathrm{~nm}$ (on-wafer) resolution. As a complementary method, the a-priori knowledge of the sample is employed to identify potential defect sites by analyzing the diffraction patterns. In this method, the recorded diffraction patterns are compared with the die or database data (i.e. previously measured or calculated diffraction data from the defect-free mask layout respectively) and their difference is interpreted as the defect signal. Dynamic software filtering helps to suppress the strong diffraction from defect-free structures and allows registration of faint defects with high sensitivity. Here, we discuss the basic principles of these Fourier domain techniques and its potential for actinic mask inspection with high signal-to-noise ratio and high throughput.
\end{abstract}

Keywords: EUV lithography, actinic inspection, defect inspection, lensless imaging, mask metrology, coherent diffraction

\section{INTRODUCTION}

As EUV lithography is gathering momentum and being pitched for introduction into high-volume manufacturing, pieces of the EUVL infrastructure are under preparation. In the purview of mask metrology readiness, blank inspection and actinic mask review tools are being developed. Nevertheless, one of the key technology of EUVL metrology infrastructure, a tool for actinic patterned mask inspection, is missing. For the realization of defect-free EUV photomasks, there is a general consensus for the need for integral solutions for actinic patterned mask inspection as well as for the final through-pellicle inspection.

Research and development in actinic metrology tools have been very intensive in the last decade and various tools have been demonstrated. These tools differ in their concepts (detection and imaging modalities), illumination source (coherent or incoherent photons), and in the optics employed [1-4]. Berkeley's tool, the SHARP, is the leading research tool [5, 6] and uses coherent EUV illumination in conjunction with an off-axis Fresnel zone plate (FZP) and presently provides an ultimate resolution of $26 \mathrm{~nm}$. It is a very powerful research tool for mask review which has significant contributions to EUV mask research. The AIMS prototype, developed by Carl Zeiss, is currently the only actinic review tool commercially available [7].

In addition to lens-based microscopy, coherent diffraction imaging (CDI) methods have emerged as a promising alternative for EUV mask metrology. Various CDI techniques have been successfully used for actinic metrology where the tools differ in concepts, purpose, source, detection, and optics. The EUV group at the New Subaru, Japan, is developing a CDI-based EUV mask inspection tool [8]. In Hanyang University, a stand-alone tool based on CDI method

Photomask Technology 2016, edited by Bryan S. Kasprowicz, Peter D. Buck, Proc. of SPIE Vol. 9985, 99851P · C 2016 SPIE · CCC code: 0277-786X/16/\$18 · doi: 10.1117/12.2242961 
is being developed [9] for CD analysis of patterned masks. These aforementioned CDI methods are only limited to periodic patterns, unlike scanning CDI methods, such as ptychography, which can reconstruct arbitrary patterns. Scanning CDI has been demonstrated by several groups at EUV [10-13], showing the increasing interest in the method. Contrary to scanning microscopy and full-field microscopy, where the resolution is limited by the spot size or the NA of the lens, the achievable resolution with this method depends on signal-to-noise and NA of the detector. Recently, we have achieved $40 \mathrm{~nm}$ resolution [14], demonstrating the potential of the method for actinic imaging of EUV photomasks.

Driven by the fact that CDI is a promising method and to date there is no proven concept (research phase or commercial) that achieves the targeted throughput for patterned mask inspection, we, at PSI, have started a R\&D program towards developing an integrated actinic tool solution. Here we review the progress and discuss the outlook. We present our recent results in CDI for high-resolution imaging and show the potential of scanning scattering contrast microscopy for high-throughput defect inspection.

\section{LENSLESS METROLOGY}

Lensless metrology or Fourier-domain optical methods, refer to the observation of a sample in its Fourier domain. In these techniques, instead of a lens, a detector is placed to capture the scattered light from the sample. The scattering pattern, at a certain distance from the sample where Fraunhofer approximation is valid, is proportional to the Fourier transform of the aerial image of the sample. Figure 1 shows the measurement scheme of a generic lensless metrology method. In classical metrology methods, e.g. CD-SAXS (critical dimension measurement with small angle X-ray scattering), spatial frequency information of the sample, such as average CD or line edge roughness (LER), is obtained. This information obtained in Fourier domain (reciprocal space), is averaged over the whole area of illumination and provides very accurate measurement of "mean" CD and LER, whereas all the detailed local information of the sample is lost. This is due to the limited coherence of the illuminating beam, resulting in smearing of the fine features of the scattering pattern if the coherence length of the light is smaller than the diameter of the illumination spot. If the spot size small or the incident light is coherent, the scattering pattern captures the fine features and corresponds to the Fourier transform of the sample. The imaging detectors record only the intensity of the incoming radiation, and thereby the phase information gets lost during the detection process. Therefore, in general, an inverse Fourier transform cannot uniquely reproduce the aerial image of the sample. This, so-called "phase problem", has been overcome by phase-retrieval methods, enabling imaging without lens. In the last decades various computational algorithms have been developed and optimized to be very efficient and effective, making lensless microscopy techniques or coherent diffraction imaging (CDI) methods widely used as effective imaging techniques in various applications and for a broad range of wavelengths. CDI offers several advantages over the conventional techniques: It does not require imaging optics and thereby significantly reduces the complexity and cost of the optical setup, circumvents resolution and depth-of-focus limitations of the optics, and has a large working distance enabling through-pellicle imaging. Moreover, with a 2D scan, both amplitude and phase maps of the aerial image are obtained, whereas in imaging with lens, through-focus scans are needed in order to obtain the phase information. On the other hand, CDI requires relatively high quality scattering patterns, namely redundancy through overlap of illumination area of adjacent acquisitions, high dynamic-range and lownoise scattering patterns, and relatively high coherence of the source. These requirements make it challenging to perform defect inspection within a short time, although the throughput might reach the target specifications in future $[14,15,16]$.

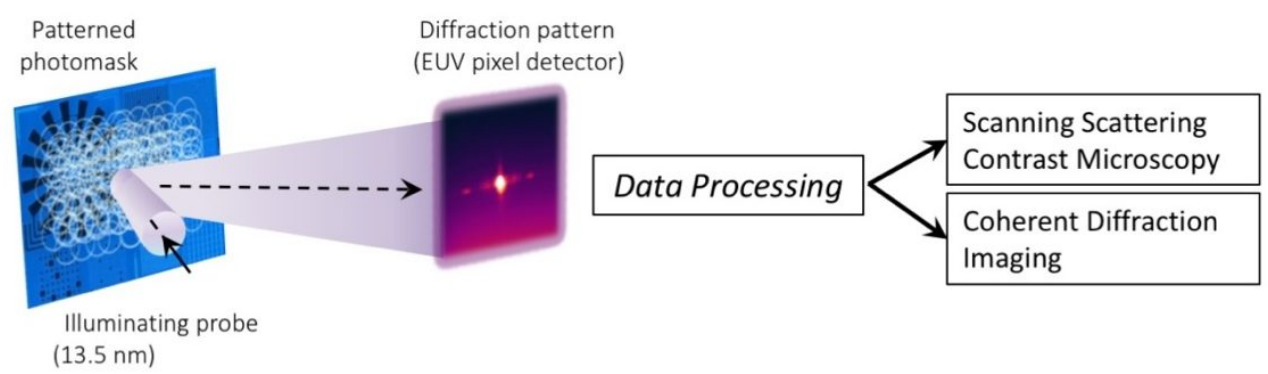

Figure 1: A schematic illustration of the concept of lensless metrology: The photomask is scanned with an illumination of finite spot size. At each location, the scattered light is collected by a pixel detector facing the photomask, and thus providing reciprocal-space (Fourier domain) information of the photomask. The collected scattering profiles can be used for (1) coherent diffraction imaging (CDI) or (2) scanning scattering contrast microscopy (SSCM). 
(a) Die/model database

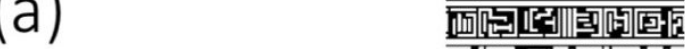

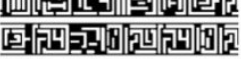

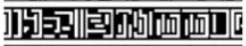

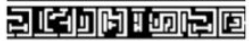

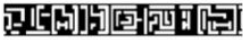

Measured scattering pattern

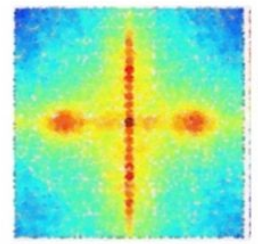

Computation
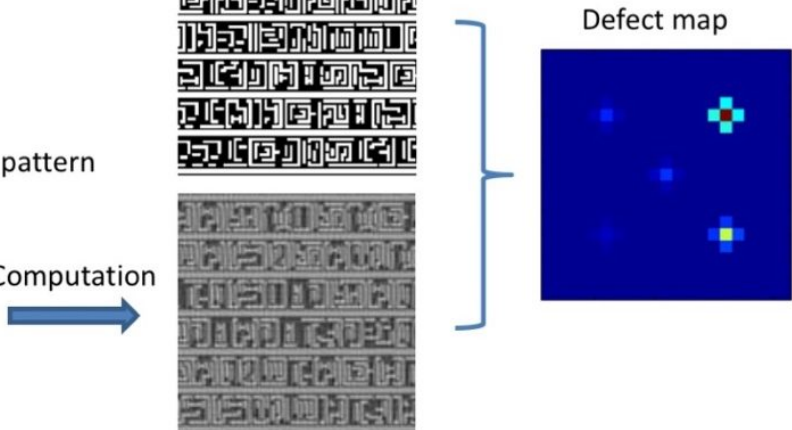

Reconstructed aerial image

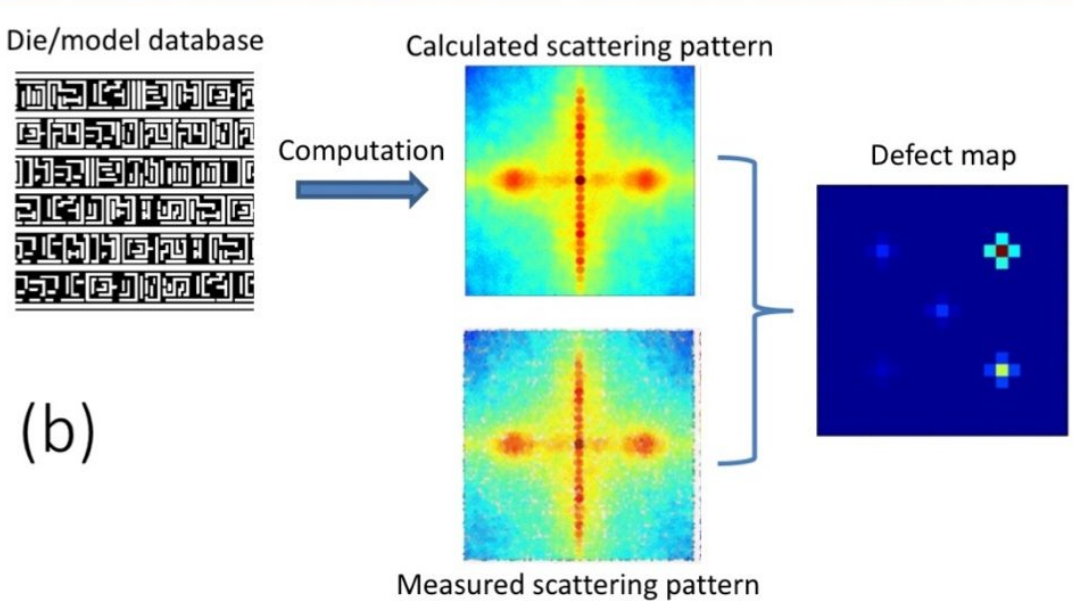

Figure 2: Defect inspection in (a) real domain and (b) Fourier domain using lensless metrology. (a) In scanning coherent diffraction imaging (CDI) measured series of scattering/diffraction patterns are used to reconstruct the aerial image of the mask. Die-to-database die-to-die comparison results in a defect map. (b) In scanning scattering contrast microscopy, the measured scattering pattern is compared with the calculated scattering pattern of the model, i.e. Fourier transform of the mask layout. A significant deviation of the measured data from the calculated data reveals the defect locations.

A promising method to increase the throughput is scanning scattering contrast microscopy (SSCM) [15]. This method is similar to CDI in terms of experimental setup and data acquisition, the difference being only in the choice of the data analysis. The comparison of CDI and SSCM is shown in Figure 2. In CDI, the scattering patterns are fed into the algorithm, which involves multiple Fourier transforms for the iterative phase retrieval and reconstructs the aerial image of the photomask. The image can be used for die-to-die or die-to-model comparison in order to obtain the defect map. In SSCM, the measured scattering pattern is directly compared either with the measured scattering pattern from another die or with the calculated scattering pattern of the mask layout. The calculated scattering pattern in this case is the Fourier transform of the modeled aerial image of the photomask. A defect is registered when there is a significant difference between the two scattering patterns. In summary, SSCM performs comparison in Fourier space whereas CDI does this in real space. The advantages of SSCM compared to CDI are higher throughput, less computational load, and higher signalto-noise ratio for the defect detection. The disadvantage of SSCM in comparison to CDI is the precision of the defect location. In SSCM, when a defect signal is detected, the spatial information of the defect is limited to the illumination spot size, or more precisely, to the overlapping regions of multiple detections. Nevertheless, a precise localization of the defect can be obtained in a straightforward manner: since the data acquisition is the same, CDI algorithms can be applied on the scattering patterns where a defect signal is detected or a slow scan can be performed at the defect site if the data quality is not sufficient. 


\section{RESCAN: ACTINIC LENSLESS METROLOGY TOOL AT PSI}

In order to demonstrate the potential of actinic lensless metrology, we have developed the RESCAN tool. The initial platform (RESCAN 1) was installed at XIL-II beamline of Swiss Light Source (SLS) [17]. Since the beamline was optimized for EUV interference lithography, neither the beam parameters nor the infrastructure was optimal for EUV lensless metrology experiments. The most severe limitation was the relatively broad bandwidth of the EUV light (4\%). We developed an optical setup with a Fresnel Zone Plate, which focuses the beam on a pinhole and thereby increase the monochromaticity of the beam up to $0.5 \%$. Figure 3 a shows the experimental setup and the details have been reported previously $[14,16]$. Using this setup, we have successfully performed CDI on EUV masks. The results and their chronological evolution over the past year are shown in Figure 4.

Encouraged with these successful early experiments, we have developed a new tool (RESCAN 2) which is design for CDI and SSCM using a monochromatic beam. We had the opportunity to obtain very limited experimental time at the SIM beamline of SLS. The schematics of the beamline and the optical layout of RESCAN 2 at SIM beamline are shown in Figure 3b. The EUV beam generated by an undulator source passes through the monochromator and is focused on a pinhole which serves as a spatial filter. The monochromatic EUV beam reaches the RESCAN chamber where it is focused with a condenser (i.e. a spherical mirror of $121 \mathrm{~mm}$ focal length) and reflected onto the photomask with a folding mirror.

Over a period of 4 months we had an experimental time of a few days per month and during this time we focused on aerial imaging using CDI of a test photomask with various random structures, a resolution test structure (Siemens star) and gratings with programmed defects. As shown in Figure 4, we have steadily improved our imaging performance in terms of resolution and removal of artifacts. Figure 4 also shows a close up actinic aerial image of a grating with $100 \mathrm{~nm}$ half-pitch with programmed defects. As seen in the figure, the grating is well resolved and averaged line profiles yielded a resolution of $40 \mathrm{~nm}$, which corresponds to $10 \mathrm{~nm}$ on wafer. This image also shows a low-frequency speckle which comes from the phase modulations due to the roughness of the multilayer, a clear indication of the sensitivity of the method.
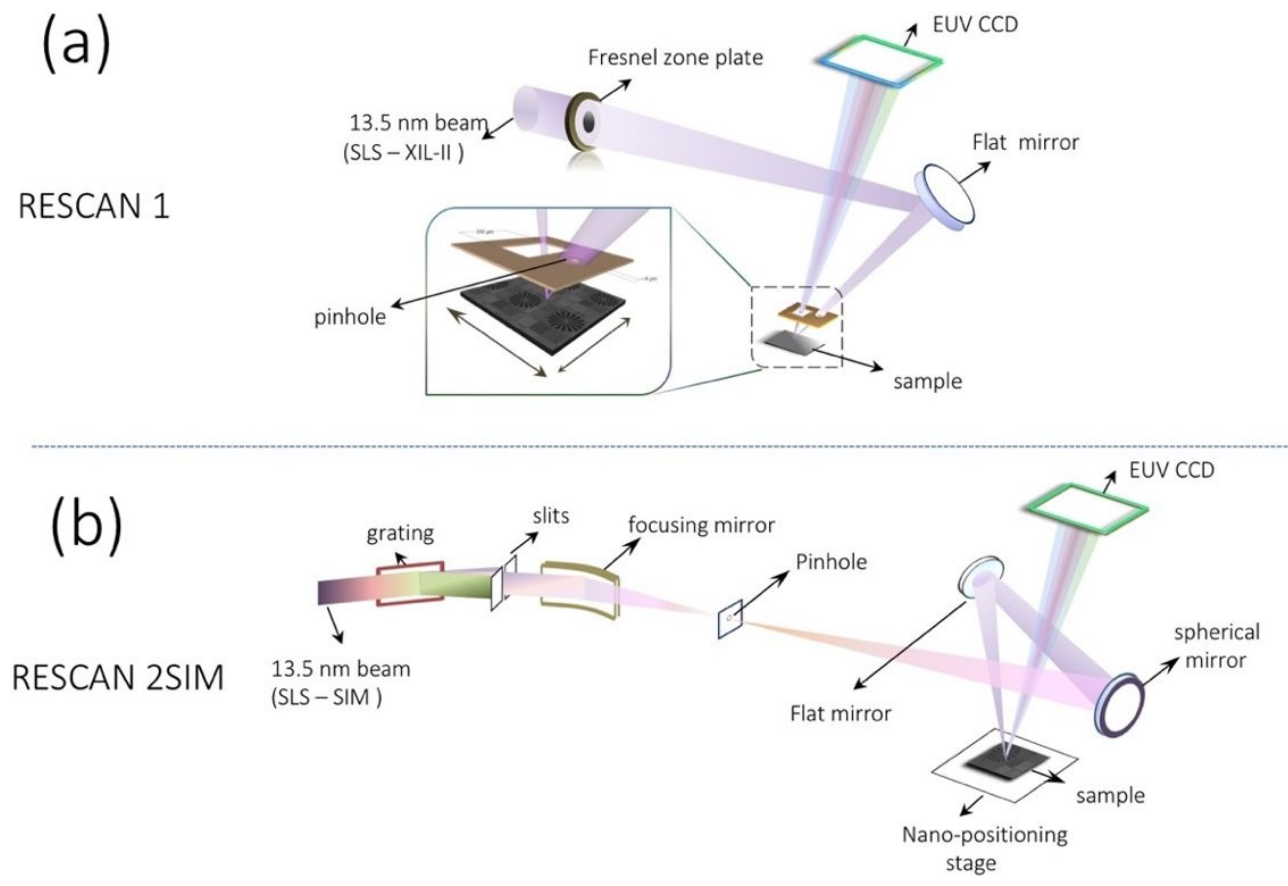

Figure 3: Schematic view of the experimental setups. (a) RESCAN-1: Setup used for the experiments at XIL beamline. EUV beam of $4 \%$ bandwidth is focused by a Fresnel zone plate (FZP) and the reflected from a multi-layer (ML) mirror through a pinhole onto the photomask. The resulting diffraction patterns are recorded on a CCD detector. Close-up view of the pinhole assembly which results in an illumination with $0.5 \%$ bandwidth. (b) RESCAN-2SIM setup with monochromatic EUV illumination. The beam is focused onto the photomask using a spherical mirror and reflected beam is recorded with a CCD. 


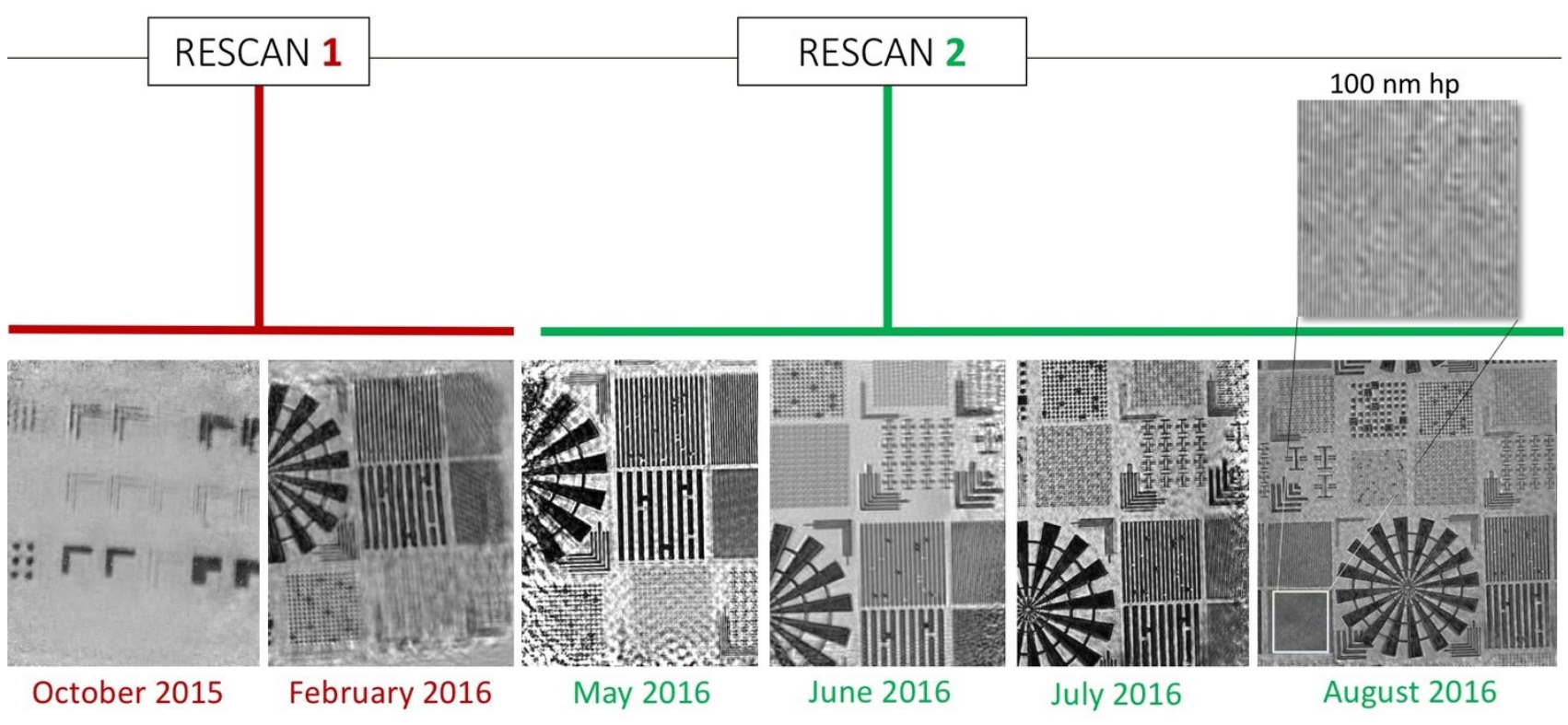

Figure 4: Experimental results of lensless imaging obtained with RESCAN 1 and RESCAN 2 tools during October 2015 to August 2016 showing the steady progress in obtaining high-resolution and artifact-free actinic aerial images. Top right: a zoom in site of the mask with a $100 \mathrm{~nm}$ grating with programmed defects.

\section{SCANNING SCATTERING CONTRAST MICROSCOPY}

As discussed in previous sections, SSCM is the most promising method to obtain a defect map with high throughput. Preliminary experimental results and initial performance estimation have been reported previously [15]. Here, we report on the predicted performance of SSCM in terms of sensitivity for contact hole masks. We have performed extensive simulations of different technology nodes and for various types of defects, using realistic detector and source conditions.

Figure 5 shows the layout of a perfect mask (A) for contact holes and its corresponding scattering pattern at the detector (B). In this case, the detector is placed at $82 \mathrm{~mm}$ above the sample. The detector parameters (with $75 \mu \mathrm{m}$ pixel size and with $50 \mathrm{e}^{-}$rms noise) are chosen from a prototype of JUNGFRAU, which is an active pixel hybrid CMOS detector developed at PSI [18]. We have confirmed these parameters also for EUV beam at our extensive detector evaluation at XIL beamline [19]. As seen in Figure 5, the scattering pattern of the mask has sharp and intense peaks, which emerge from the 2D periodicity of the mask, with a very low background. A defect will cause a measurable difference in this background. However, a realistic mask with a finite LER and surface roughness will also lead to increased background signal. This case is illustrated in Figures $5 \mathrm{c}$ and $5 \mathrm{~d}$. Our simulations have identified these as the major source of the noise, reducing the SNR of a defect's signature. In our simulations we took these realistic effects into account assuming a LER of $\sigma=1.4 \mathrm{~nm}$ with a correlation length of $\mathrm{L}_{\text {corr }}=20 \mathrm{~nm}$ and a multilayer roughness of $\sigma=50 \mathrm{pm}$. The absorber was a 75-nm-thick Tantalum Nitride $(\mathrm{TaN})$ layer. The 3D effects of the light interaction with the multilayer and the absorber are also taken into account. For the detector, a quantum efficiency of $60 \%$ at EUV wavelength and a full-frame acquisition of $2 \mathrm{kHz}$ are assumed, which are the feasible parameters for this technology. For the source, we have assumed $10 \mathrm{~mW}$ power on the mask level, which is the design specification of the high-brightness compact EUV source $[16,18]$. For our performance evaluations we have fixed the spot size a value of $40 \mu \mathrm{m}$ and a step size of $16 \mu \mathrm{m}$. This leads to the targeted throughput with scanning time of less than 6 hours for a 4"-inch patterned area of a photomask.

The type of defects that we evaluated in the present study are shown in Figure 6. The isolated defects are $20 \mathrm{~nm}$ in shortest dimension and CD errors are $8 \mathrm{~nm}$ on mask. We have repeated the simulations for the same mask layout and defects types for the N10, N7, and N5 technology nodes corresponding to $92 \mathrm{~nm}, 64 \mathrm{~nm}$, and $44 \mathrm{~nm}$ half-pitch on mask, respectively. The calculated SNR values are listed in Table 1. The SNR values are given for two different detector NA, i.e. solid angle of the scattering pattern captured by the detector. We observed that with higher NA detectors the SNR increases. This is due to the fact that more signal of a defect is captured when the acceptance angle of the detector is 
larger. As seen in the table, we observed very high SNR values for most cases. Nevertheless, in some cases the SNR values decrease below the targeted SNR value of 8 . We note that the reported SNR values refer to single detection values whereas, due to the spot size and step size specified above, each defect is detected at least 4 times in a meander scan. This gives further redundancy to the detection and increases the SNR by factor of two. We take this factor as an error margin for experimental errors, such as detector, source fluctuations, and stage inaccuracy.

A comparison of the SNR (Table 1) reveals that even for the same size defects, the SNR values can be significantly different. The major reason for this is the shadowing effect. For example, some of the intrusion defects are shadowed because of the $6^{\circ}$ oblique illumination, and have a much smaller "actinic footprint" even if they are the same size on the absorber. In conclusion, we obtained low SNR values for the highly shadowed defects, which probably will not print. We did a simple check of printability for few of the defects. The actinic aerial image of the defected mask was calculated and a blur with $30 \%$ of $\mathrm{CD}$ is added to account for the blur of a typical CAR resist. The thresholded binary image accounts for the obtained resist pattern. For the defects with $\mathrm{SNR}<10$, the resulting $\mathrm{CD}$ error in the resist pattern was less than $10 \%$, and therefore can be regarded as not printable.

In conclusion, achieving the targeted specifications of mask inspection $(<8$ hours total inspection time, SNR $>8$ for all printable defects) is possible with a suitable detector, source, and algorithms. Further analysis will be made to check the printability of the all defects with SNR $<8$. Experiments with a CCD demonstrated the proof of principle [15], in which the dynamic range of the detector was the bottleneck. In future, experiments with a high-dynamic range detector will be performed.

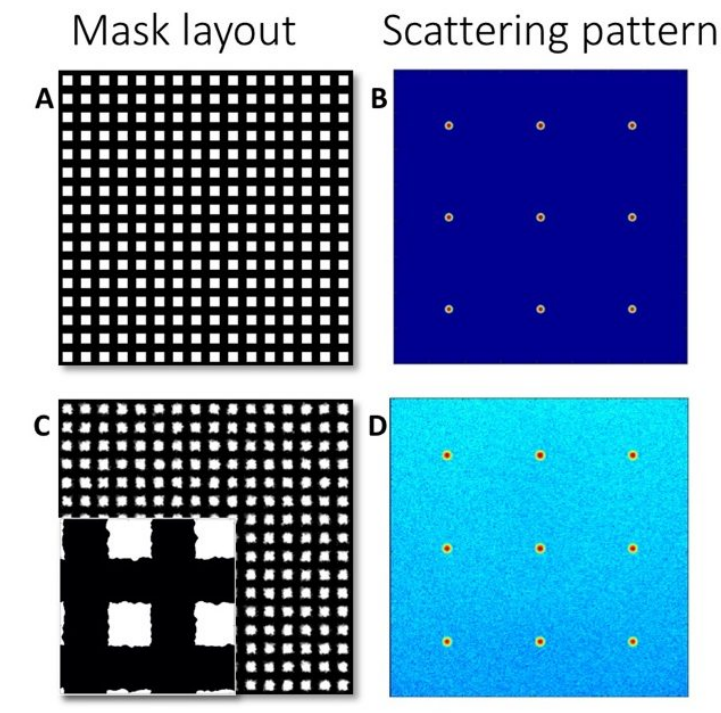

Figure 5: Layouts of a contact hole masks with no line edge roughness (A) and with a realistic line edge roughness (C) and their corresponding scattering pattern at the pixel detector. In case of a perfect mask, the scattering pattern is only the diffraction peaks of the $2 \mathrm{D}$ periodic array (B) and in case of a realistic line edge roughness, an additional signal of diffuse background is observed resulting from the roughness (D). 


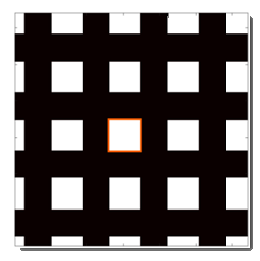

$\mathrm{CD}$ error $+8 \mathrm{~nm}$

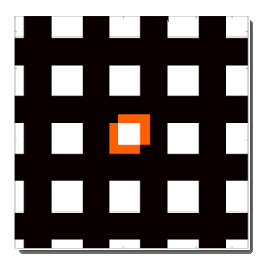

Hole shilt

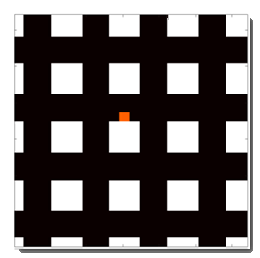

Intrusion (up)

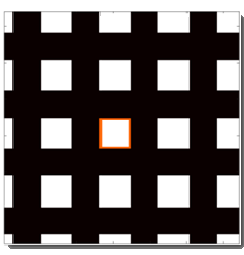

CD error $-8 \mathrm{~nm}$

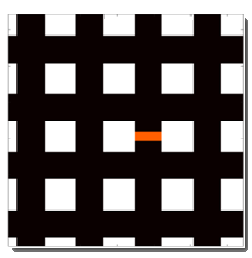

Bridge (hor izorlal)

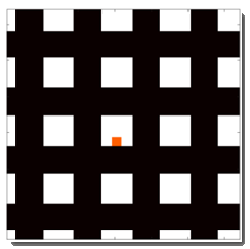

Extrusion(dow))

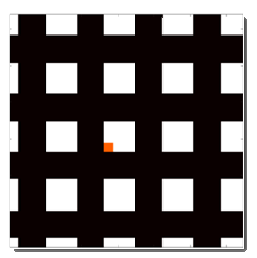

Chiro ịdown)

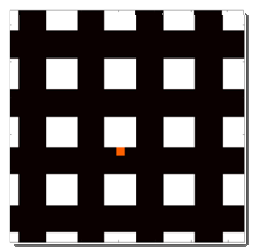

Inliusionịdown)

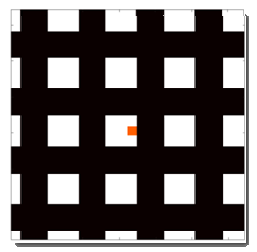

Extrusion (horizontal)

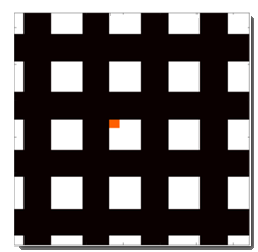

Chirp(up)

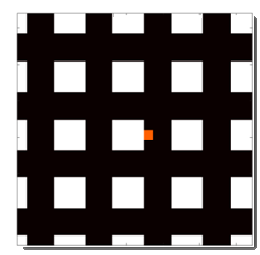

Intrusion (horizontal)

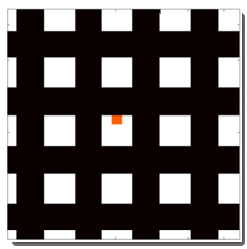

Extrusion (up)

Figure 6: Different types of absorber defects evaluated in this study. All the point defects are $20 \mathrm{~nm}$ in the smallest dimension and CD errors are $8 \mathrm{~nm}$. The hole shift is also $20 \mathrm{~nm}$.

Table 1: SNR ratios of defect signals in scattering contrast microscopy for the contact hole mask and different defects (described in Figure 6). The SNR values are given for two different detector NA, i.e. solid angle of the scattering pattern captured by the detector. The technology nodes of N10, N07 and N05 correspond to $92 \mathrm{~nm}, 64 \mathrm{~nm}$, and $44 \mathrm{~nm}$ half-pitch on mask.

\begin{tabular}{|c|c|c|c|c|c|c|c|}
\hline Defect name & \multicolumn{2}{|c|}{ SNR (NA $=\mathbf{0 . 3 3}$} & \multicolumn{3}{c|}{ SNR (NA = 0.41) } \\
\hline & N05 & N07 & N10 & N05 & N07 & N10 \\
\hline CD error & 16.5 & 19.7 & 28.9 & 20.6 & 25.5 & 37.5 \\
\hline CD error & 14.8 & 18.2 & 26.7 & 19.1 & 22.6 & 36.0 \\
\hline Shift & 123.9 & 233.5 & 415.0 & 177.4 & 314.3 & 507.6 \\
\hline Intrusion (down) & 8.4 & 4.3 & 5.0 & 9.8 & 4.9 & 6.7 \\
\hline Intrusion (horizontal) & 3.8 & 4.1 & 5.7 & 5.3 & 6.5 & 8.7 \\
\hline Intrusion (up) & 11.7 & 13.5 & 10.1 & 19.1 & 23.6 & 17.6 \\
\hline Extrusion (down) & 16.0 & 14.4 & 10.4 & 17.5 & 15.1 & 11.3 \\
\hline Extrusion (horizontal) & 22.0 & 17.2 & 22.5 & 28.3 & 22.4 & 29.8 \\
\hline Extrusion (up) & 38.0 & 43.5 & 41.8 & 57.2 & 64.8 & 62.2 \\
\hline Chirp (down) & 6.5 & 6.8 & 7.4 & 7.7 & 8.5 & 9.3 \\
\hline Chirp (up) & 17.0 & 15.3 & 13.6 & 27.3 & 25.1 & 22.7 \\
\hline \multicolumn{2}{|c|}{} & & & &
\end{tabular}




\section{CONCLUSION AND OUTLOOK}

The method we outlined, based on SSCM and CDI, can provide a powerful and effective tool for actinic defect inspection by overcoming the limitations of EUVoptics. All the necessary hardware components are either available or feasible. We showed the feasibility of mask inspection with targeted specifications through extensive simulations. Further optimization of the experimental parameters, such as detector distance, spot size, step size, illumination NA, and more advanced algorithms will probably increase the SNR values further. We are currently commissioning an upgraded version of the RESCAN tool that we will use to demonstrate the feasibility of lensless actinic defect inspection in practice. This dedicated setup will significantly increase the speed of our R\&D efforts. This tool will be test bed for further instrumentation and algorithm testing and it will be a significant step towards realization of a stand-alone tool.

\section{REFERENCES}

[1] K. A. Goldberg, I. Mochi, "Wavelength-Specific Reflections: A Decade of EUV Actinic Mask Inspection Research,” J. Vac. Sci. Technol. B 28, C6E1 (2010).

[2] K. A. Goldberg, M. P. Benk, A. Wojdyla, E. Verduijn, O. R. Wood, P. Mangat," EUV actinic brightfield mask microscopy for predicting printed defect images," Proc. SPIE 9635, Photomask Technology 2015, 963514 (2015).

[3] T. Haga, H. Kinoshita, K. Hamamoto, S. Takada, N. Kazui, S. Kakunai, H. Tsubakino, T. Watanabe, "Evaluation of Finished Extreme Ultraviolet Lithography (EUVL) Masks Using a EUV Microscope,” Jpn. J. Appl. Phys. 42, 3771 (2003).

[4] L. Bahrenberg, S. Herbert, J. Tempeler, A. Maryasov, O. Hofmann, S. Danylyuk, R. Lebert, P. Loosen, L. Juschkin, "Analysis of distinct scattering of extreme ultraviolet phase and amplitude multilayer defects with an actinic darkfield microscope," Proc. SPIE 9422, Extreme Ultraviolet (EUV) Lithography VI, 942229 (2015)

[5] K. A. Goldberg, I. Mochi, S. B. Rekawa, N. S. Smith, J. B. Macdougall, P. P. Naulleau, "An EUV Fresnel zoneplate mask-imaging microscope for lithography generations reaching 8 nm," Proc. SPIE 7969, 796910 (2011).

[6] K. A. Goldberg, I. Mochi, M. Benk, A. P. Allezy, M. R. Dickinson, C. W. Cork, D. Zehm, J. B. Macdougall, E. Anderson, F. Salmassi, W. L. Chao, V. K. Vytla, E. M. Gullikson, J. C. DePonte, M. S. G. Jones, D. Van Camp, J. F. Gamsby, W. B. Ghiorso, H. Huang, W. Cork, E. Martin; E. Van Every, E. Acome, V. Milanovic, R. Delano, P. Naulleau, S. B. Rekawa, "Commissioning an EUV mask microscope for lithography generations reaching $8 \mathrm{~nm}$," Proc. SPIE 8679, Extreme Ultraviolet (EUV) Lithography IV, 867919 (2013).

[7] D. Hellweg, S. Perlitz, K. Magnusson, R. Capelli, M. Koch, M. Malloy, "Actinic review of EUV masks: performance data and status of the AIMS EUV System," Proc. SPIE 9776, Extreme Ultraviolet (EUV) Lithography VII, 97761A (2016).

[8] T. Harada, J. Kishimoto, T. Watanabe, H. Kinoshita, D. G. Lee, "Mask observation results using a coherent extreme ultraviolet scattering microscope at New SUBARU," J. Vac. Sci. Technol. B 27, 3203 (2009).

[9] S. Lee, J. G. Doh, J. U. Lee, I. Lee, C. Y. Jeong, D. G. Lee, S. Rah, J. Ahn, "Carbon contamination of EUV mask and its effect on CD performance," Current Appl. Phys. 11, S107 (2011).

[10] J. Bussmann, M. Odstrčil, R. Bresenitz, D. Rudolf, J. Miao, W. S. Brocklesby, D. Grützmacher, L. Juschkin, "Employing partially coherent, compact gas-discharge sources for coherent diffractive imaging with extreme ultraviolet light," Proc. SPIE 9589, X-Ray Lasers and Coherent X-Ray Sources: Development and Applications XI, 95890L (2015)

[11] M. Odstrcil, J. Bussmann, D. Rudolf, R. Bresenitz, Jianwei Miao, W. S. Brocklesby, L. Juschkin, "Ptychographic imaging with a compact gas-discharge plasma extreme ultraviolet light source," Optics Letters, 40(23), pp. 5574$5577(2015)$

[12] T. Harada, H. Hashimoto, T. Amano, H. Kinoshita, T. Watanabe, "Phase imaging results of phase defect using micro-coherent extreme ultraviolet scatterometry microscope,“ J. Micro/Nanolith. MEMS MOEMS. 15(2), 021007 (2016).

[13] B. Zhang, D. F. Gardner, M.D. Seaberg, E. R. Shanblatt, H. C. Kapteyn, M. M. Murnane, D. E. Adams, "High contrast 3D imaging of surfaces near the wavelength limit using tabletop EUV ptychography," Ultramicroscopy 158, 98 (2015).

[14]P. Helfenstein, I. Mohacsi, R. Rajendran, Y. Ekinci, "Scanning coherent diffractive imaging methods for actinic EUV mask metrology," Proc. SPIE 9776, 97761F (2016).

[15] I. Mohacsi, P. Helfenstein, R. Rajendran, Y. Ekinci, "Scanning scattering contrast microscopy for actinic EUV mask 
inspection," Proc. SPIE 9778, 977810 (2016).

[16] P. Helfenstein, I. Mohacsi, R. Rajendran, Y. Ekinci, "Scanning coherent diffractive imaging methods for actinic EUV mask metrology," J. Micro/Nanolith. MEMS MOEMS 15(3), 034006 (2016).

[17] N. Mojarad, J. Gobrecht, Y. Ekinci, "Interference lithography at EUV and soft X-ray wavelengths: Principles, methods, and applications," Microelectron. Eng. 143, 55-63 (2015).

[18] J. H. Jungmann-Smith, A. Bergamaschi, S. Cartier, R. Dinapoli, D. Greiffenberg, I. Johnson, D. Maliakal, D. Mezza, A. Mozzanica, C. Ruder, L. Schaedler, B. Schmitt, X. Shi, G. Tinti, "JUNGFRAU 0.2: prototype characterization of a gain-switching, high dynamic range imaging system for photon science at SwissFEL and synchrotrons," Journal of Instrumentation 9, 12013 (2014).

[19] To be published. 